\title{
Composição dos Resíduos Poliméricos, Pós-consumo, Gerados no Município de São Carlos, SP
}

\author{
Tássio F. L. Matos \\ Centro de Pesquisa em Resíduos Sólidos, CEFET, CE, \\ PPG-SEA, EESC, USP
}

\author{
Valdir Schalch \\ Núcleo de Estudo e Pesquisa em Resíduos Sólidos, PPG-SEA, EESC, USP
}

\begin{abstract}
Resumo: Os resíduos poliméricos pós-consumo - embalagens plásticas - se destacam nos resíduos sólidos domiciliares, por apresentarem crescimento de participação no lixo urbano e possuírem características como degradação lenta e volumetria elevada, o que compromete a vida útil dos aterros sanitários. Ressalta-se, ainda, o potencial econômico para reutilização e reciclagem dos resíduos poliméricos. Este trabalho tem por objetivo apresentar a composição percentual, mássica, dos resíduos poliméricos no município de São Carlos, SP. O método adotado para caracterização, para determinação da composição, foi por amostragem, na coleta regular, e pela massa total coletada, na coleta seletiva. Na coleta regular, o número de amostragem compreendeu todos os quinze setores, sendo a massa da amostra obtida por quarteamento. Foi feita uma caracterização no inverno e outra no verão. Destacam-se, nos resultados da coleta regular, os percentuais em massa de 10,47\% de resíduos poliméricos, o PET com maior participação $(35,96 \%)$ nas resinas e o uso preferencial em embalagens para alimentação $(56,42 \%)$, e, na coleta seletiva, os percentuais de 20,60\% de resíduos poliméricos, a resina PET com maior participação $(50,64 \%)$ e uso preferencial em embalagens para alimentação $(66,06 \%)$. Os resultados evidenciam a tendência de crescimento de resíduos de PET e do uso preferencial dos polímeros termoplásticos em embalagens para alimentação.
\end{abstract}

Palavras-chave: Polímeros, resíduos poliméricos pós-consumo, resíduos plásticos, caracterização.

\section{Composition of Post-consumption Polymeric Wastes Generated in the Municipality of São Carlos, SP}

Abstract: Polymeric wastes have become important in household waste, with increasing contribution to the urban waste. They present the following characteristics: slow degradation and high volume, which compromises the life cycle of sanitary landfills, and may also have economical potential in the re-use and recycle. The objective of this work is to present a characterization of the polymeric wastes conducted in the conventional and selective collection of the municipality of São Carlos, SP. The method adopted for characterization in regular collection was sampling, while for the selective collection we employed the total weight collected. In regular collection, the number of sampling involved all the 15 sectors, being the sample mass obtained with the quarter's method. One characterization was done in the winter and another in the summer. Striking results in the regular collection were the weight percentage of $10.47 \%$ of polymeric wastes, with PET having the largest contribution (35.96\%) in the resins and the preferential use in packing for food (56.42\%). In the selective collection, the percentage polymeric waste was $20.60 \%$, with the resin PET with largest contribution (50.64\%) and preferential use in packing for food $(66.06 \%)$. The results demonstrate the growth tendency of wastes of PET and of the preferential use of the thermoplastic polymers in food package.

Keywords: Polymer, polymeric post-consumption waste, plastic waste, characterization.

\section{Introdução}

Os resíduos poliméricos pós-consumo - embalagens plásticas - se destacam nos resíduos sólidos domiciliares (RSD), pois, nas últimas décadas, têm apresentado crescimento de participação na composição do lixo urbano, conforme pode ser observado na Tabela 1. A importância de seu estudo fundamenta-se em características importantes como a degradação lenta, comprometendo a vida útil dos aterros sanitários, por apresentarem vários tipos com propriedades físicas e químicas diferentes entre si, dificultando a identifi- cação e seleção destes resíduos e, ainda, por terem elevado potencial econômico para reutilização e reciclagem.

Os polímeros sintéticos termoplásticos, ou simplesmente plásticos, como são mais conhecidos, representam a maioria dos resíduos poliméricos presentes nos resíduos sólidos domiciliare $\mathrm{S}^{[1]}$. Os resíduos poliméricos, pós-consumo, são compostos, basicamente, pelas resinas de PET (politereftalato de etileno), PEAD (polietileno de alta densidade), PEBD (polietileno de baixa densidade), PVC (policloreto de vinila), PP (polipropileno), e PS (poliestireno). 
Tabela 1. Composição, em porcentagem de peso, dos RSD de algumas cidades.

\begin{tabular}{lccrrrrc}
\hline Cidade & Ano publicação & Plástico & Vidro & Metal & Papel & Matéria orgânica & Outros \\
\hline São Carlos (SP) & 1975 & 7,2 & 6,3 & 5,6 & 20,5 & 40,4 & 20,0 \\
Rio de Janeiro (RJ) & 1986 & 9,7 & 3,4 & 3,7 & 38,4 & 38,6 & 6,2 \\
Columbia (USA) & 1987 & 7,0 & 4,0 & 6,0 & 41,0 & 7,0 & 35,0 \\
São Carlos (SP) & 1989 & 8,5 & 1,4 & 5,4 & 21,3 & 56,7 & 6,7 \\
EPA (USA) & 1994 & 12,0 & 6,0 & 6,0 & 33,0 & 9,0 & 34,0 \\
Columbia (USA) & 1996 & 16,0 & 3,0 & 6,0 & 41,0 & 9,0 & 25,0 \\
Rio de Janeiro (RJ) & 2002 & 17,61 & 2,74 & 1,97 & 18,78 & 55,96 & 2,94 \\
\hline
\end{tabular}

Fonte: Adaptado de Gomes $^{[2]}$, Zeng ${ }^{[3]}$ e COMLURB ${ }^{[4]}$.

Tabela 2. Composição de plásticos rígidos em cidades brasileiras.

\begin{tabular}{llccccccc}
\hline \multicolumn{1}{c}{ Cidade } & $\begin{array}{c}\text { Ano de realização/ } \\
\text { publicação }\end{array}$ & PEAD (\%) & PEBD (\%) & PVC (\%) & PET (\%) & PP (\%) & PS (\%) & Outros (\%) \\
\hline Araraquara (SP) & $1995 / 1996$ & 46,7 & 0,5 & 5,5 & 34,5 & 9,2 & 3,0 & 0,6 \\
Botucatu (SP) & $1997 / 2000$ & 20,4 & 4,6 & 2,4 & 33,3 & 6,2 & 33,1 & 33,1 \\
Araraquara (SP) & $1997 / 98$ e 99/2001 & 28,1 & 0,3 & 2,1 & 63,6 & 3,2 & 1,2 & 1,5 \\
\hline
\end{tabular}

Fonte: Adaptado de Zanin e Mancini ${ }^{[5]}$.

Este trabalho é justificado pela possibilidade de gerar informações referentes aos resíduos sólidos poliméricos termoplásticos, mais especificamente quanto às suas características e composições percentuais presentes nos RSD de um município brasileiro de médio porte, as quais são importantes para os aspectos sanitário e social. Para o poder público, as informações são importantes para o estabelecimento e aprimoramento de políticas públicas e a gestão integrada dos resíduos sólidos, notadamente pelos aspectos de prevenção da poluição com base na redução, reutilização e reciclagem dos plásticos. Pelo aspecto da reciclagem, os dados gerados podem fornecer a quantidade de material que pode ser separado de cada resíduo, permitindo verificar a viabilidade econômica de um processo. Outro fator importante é a constatação do crescimento constante da geração de resíduos plásticos pós-consumo, conforme pode ser observado na Tabela 1 . Na Tabela 2 pode-se observar algumas caracterizações de plásticos rígidos em cidades brasileiras.

Este trabalho tem por objetivo apresentar a composição dos resíduos poliméricos, pós-consumo, presentes na coleta regular e seletiva do município de São Carlos, SP, compreendendo a participação percentual mássica por: a) plásticos rígidos e filmes; b) tipo de resina do plástico rígido; c) uso do plástico rígido.

\section{Experimental}

\section{Área de estudo}

O município de São Carlos localiza-se na região central do Estado de São Paulo e tem população de 214.786 habitantes, estimada em $01 / 07 / 2005^{[6]}$, ano de realização desta pesquisa. O município possui sistema de coleta regular e seletiva dos RSD, assim como aterro sanitário estruturado.

A coleta regular é realizada em toda área urbana do município de São Carlos, inclusive nos distritos de Água Ver- melha e Santa Eudóxia, com estrutura logística dividida em 15 setores. Cada setor tem produção diferenciada, variando aproximadamente de 7 a 12 t de RSD, por $\mathrm{dia}^{[1]}$. Em 2005 o recolhimento diário médio de RSD, foi de aproximadamente 137 toneladas $^{[7]}$, o que representa uma produção per capita de $0,64 \mathrm{~kg} / \mathrm{hab} /$ dia da população urbana. Toda a produção de RSD da coleta regular é destinada ao aterro sanitário do próprio município.

A coleta seletiva de resíduos recicláveis foi implantada no município de São Carlos em junho de 2002 e, atualmente, apresenta uma evolução significativa com números importantes ${ }^{[8]}$, os quais se destacam:

- É realizada por três cooperativas de catadores (Ecoativa, Coopervida, Cooletiva), em modelo de autogestão, envolvendo mais de cinqüenta trabalhadores cooperados;

- A área de abrangência atinge $60 \%$ da área urbana, com aproximadamente 32.000 pontos de coleta;

- A frequiência de coleta, em cada bairro, é de uma vez por semana; e

- As cooperativas recolhem, em média, $80 \mathrm{t}$ por mês, de resíduos recicláveis, o que equivale a aproximadamente $2.700 \mathrm{~kg}$, por dia. Este montante ainda é pouco, considerando que representa cerca de $2,0 \%$ da massa coletada diariamente na coleta regular.

\section{Procedimento de caracterização}

O método de caracterização para determinação dos resíduos poliméricos, adotado neste trabalho, tem fundamentação na metodologia presente em Gomes ${ }^{[2]}$, com adaptação e atualização dos procedimentos conforme orientações do Instituto de Pesquisa Tecnológica do Estado de São Paulo (IPT) em publicação conjunta com o Compromisso Empresarial para a Reciclagem (CEMPRE) ${ }^{[9]}$, e da Associação Brasileira de Normas Técnicas $(A B N T)^{[10]}$. Para verificação da influência climática foi realizada uma caracterização no inverno e outra no verão. 


\section{Preparação da amostra na coleta regular}

Para maior significância do número de amostragem optou-se em caracterizar todos os quinze setores da coleta regular, abrangendo, portanto, toda a área urbana.

Considerando a expectativa de geração elevada de resíduos sólidos domiciliares, em cada setor, tornou-se conveniente a adoção de um procedimento para obtenção de amostra significativa da massa de resíduos coletados. Segundo KLEE (1980 apud ZENG ${ }^{[3]}$, tradução nossa):

[...] quando os pesos da amostra diminuem abaixo de aproximadamente de $91 \mathrm{~kg}$, aumenta, rapidamente, a discrepância na amostra de lixo, mas que acima de aproximadamente $140 \mathrm{~kg}$, a discrepância aumenta lentamente [...] recomenda um peso de amostra entre 91 e $140 \mathrm{~kg}$.

O intervalo de 91 a $140 \mathrm{~kg}$, para o peso das amostras de resíduos sólidos é recomendado, também, pela American Society For Testing And Materials (ASTM) ${ }^{[11]}$.

Neste trabalho foi adotada a massa aproximada de $200 \mathrm{~kg}$, obtida após quarteamento, conforme o procedimento para preparação da amostra a ser caracterizada, o qual teve os seguintes passos:

- Coleta de aproximadamente $400 \mathrm{~kg}$ de lixo na pilha descarregada pelo caminhão coletor, de cada setor, em área reservada no aterro sanitário de São Carlos/SP. Essa massa foi aferida em balança marca Toledo do Brasil, modelo 2095 dd/IU, e disposta sobre uma lona plástica de $8 \times 10 \mathrm{~m}$;

- Dividiu-se a amostra em quatro montes de $100 \mathrm{~kg}$ e aplicou-se o processo de quarteamento em cada monte. Terminado o processo de quarteamento juntaram-se as partes reservadas para a seleção dos resíduos, conforme a Figura 1; e

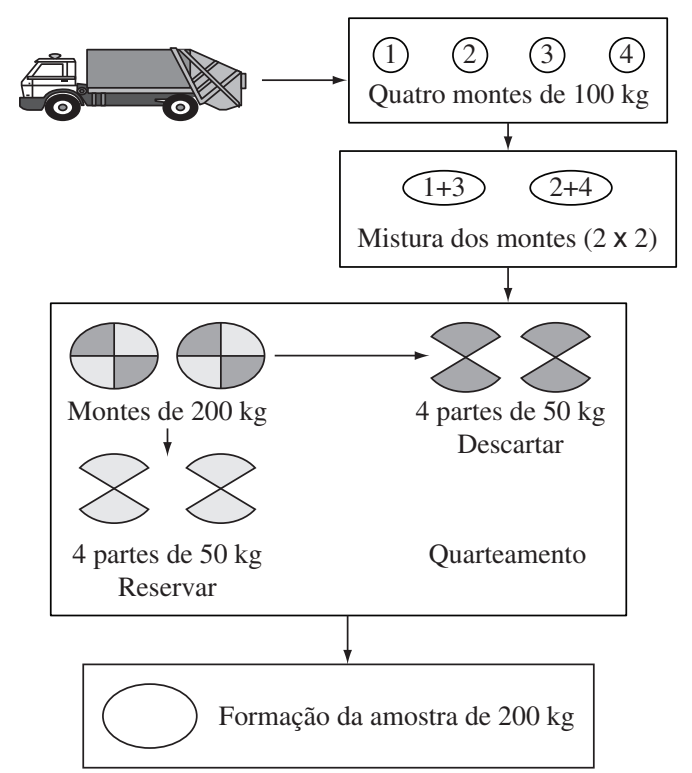

Figura 1. Diagrama de preparação da amostra de RSD da coleta regular.
- A amostra resultante foi submetida à caracterização por meio da separação e aferição da massa dos resíduos sólidos domiciliares (papel/papelão, vidro, embalagem longa vida, metal, rejeito, plástico rígido e plástico filme). Os plásticos rígidos foram reservados para caracterização por tipo de resina e uso.

\section{Preparação da amostra na coleta seletiva}

$\mathrm{Na}$ coleta seletiva a caracterização foi feita na massa total dos resíduos recicláveis coletados, não havendo necessidade de estabelecer um procedimento de amostragem.

A preparação da amostra consistiu na adaptação da organização dos trabalhos das cooperativas e se deu, portanto, da seguinte forma:

- Obtenção da massa dos resíduos recicláveis coletados em cada dia programado para caracterização; e

- Separação e aferição da massa dos resíduos recicláveis (papel/papelão, vidro, embalagem longa vida, metal, rejeito, plástico rígido e plástico filme) presentes na massa total coletada de cada dia. Os plásticos rígidos foram reservados para caracterização por tipo de resina e uso.

\section{Caracterização dos resíduos poliméricos - plásticos rígidos}

$\mathrm{O}$ procedimento de caracterização dos plásticos rígidos foi o mesmo nos dois sistemas de coleta.

A massa da amostra de plásticos rígidos foi submetida ao processo de identificação e seleção por tipo de resina polimérica termoplástica e por tipo de uso, conforme o diagrama de seleção hierárquica apresentado na Figura 2.

Para a identificação do tipo de polímero foram utilizados os seguintes procedimentos:

- Identificação por codificação, conforme a Tabela 3, presente na embalagem. A maioria dos resíduos foi identificada por este método; e

- Identificação por teste comparativo de característica e propriedade, o qual foi utilizado, poucas vezes, em resíduos que não tinham o código de identificação, e apenas para distinguir os resíduos de PEAD e PP. Para diferenciação entre os resíduos de PEAD e PP foram empregados os testes seguintes: Correlação com produtos cujas

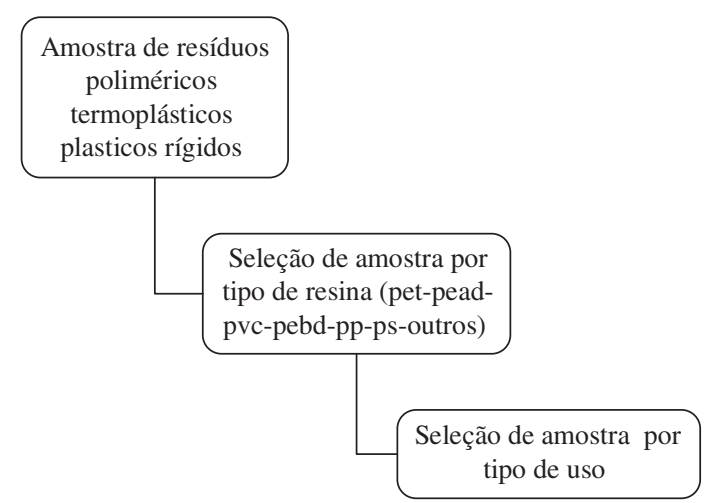

Figura 2. Diagrama da seleção hierárquica dos resíduos poliméricos. 
Tabela 3. Códigos e Siglas de identificação dos polímeros sintéticos.

\begin{tabular}{cll}
\hline Código & \multicolumn{1}{c}{ Sigla } & \multicolumn{1}{c}{ Polímero } \\
\hline 1 & PET & Poli (etileno tereftalato) \\
2 & PEAD & Polietileno de alta densidade \\
3 & PVC & Poli (cloreto de vinila) \\
4 & PEBD & Polietileno de baixa densidade \\
5 & PP & Polipropileno \\
6 & PS & Poliestireno \\
7 & Outros & - \\
\hline
\end{tabular}

Fonte: $\mathrm{ABNT}^{[12]}$

embalagens geralmente utilizam polímeros conhecidos e comportamento mecânico como dureza (os resíduos de PEAD eram identificados pela facilidade de serem riscados superficialmente) e embranquecimento na dobra (os resíduos de PP eram identificados por apresentarem embranquecimento quando dobrados).

\section{Resultados e Discussão}

Os resultados obtidos compreendem as participações percentuais médias, em massa, por estação climática, nas duas coletas, dos plásticos rígidos e filmes, apresentados na Tabela 4, das resinas poliméricas dos plásticos rígidos, presentes na Tabela 5 e dos tipos de uso dos polímeros, mostrados na Tabela 6.

Foi determinado que os resíduos poliméricos, pós-consumo, da coleta regular do município de São Carlos, SP, apresentam percentual de $10,47 \%$ da massa de RSD, com composição de $2,84 \%$ de plásticos rígidos e $7,63 \%$ de plásticos filmes. Na coleta seletiva, os resíduos poliméricos têm percentual de $20,60 \%$ da massa de resíduos recicláveis, com composição de $17,02 \%$ de plásticos rígidos e 3,58\% de plásticos filmes. Em números absolutos, as cooperativas coletam, por dia, aproximadamente $556 \mathrm{~kg}$ de resíduos poliméricos, desse total, cerca de $460 \mathrm{~kg}$ são de plásticos rígidos e $96 \mathrm{~kg}$ de plásticos filmes. A coleta regular destina ao aterro sanitário, diariamente, aproximadamente $14.340 \mathrm{~kg}$ de resíduos poliméricos, desse total, cerca de $3.900 \mathrm{~kg}$ são de plásticos rígidos e $10.440 \mathrm{~kg}$ de plásticos filmes. Estes resultados mostram que a participação percentual dos resíduos poliméricos destinados ao aterro, ainda é significativa, mesmo o município possuindo sistema de coleta seletiva em $60 \%$ da área urbana.

Tabela 4. Composição de resíduos poliméricos - plástico rígido e plástico filme - por tipo de plástico, gerados no município de São Carlos, SP, em percentual de massa.

\begin{tabular}{|c|c|c|c|c|c|c|c|c|c|c|c|c|}
\hline \multirow{3}{*}{$\begin{array}{c}\text { Coleta } \\
\text { Estação } \\
\text { Tipo de plástico }\end{array}$} & \multicolumn{6}{|c|}{ Regular } & \multicolumn{6}{|c|}{ Seletiva } \\
\hline & \multicolumn{2}{|c|}{ Inverno } & \multicolumn{2}{|c|}{ Verão } & \multicolumn{2}{|c|}{ Média geral } & \multicolumn{2}{|c|}{ Inverno } & \multicolumn{2}{|c|}{ Verão } & \multicolumn{2}{|c|}{ Média geral } \\
\hline & Rígido & Filme & Rígido & Filme & Rígido & Filme & Rígido & Filme & Rígido & Filme & Rígido & Filme \\
\hline Média & 2,76 & 6,43 & 2,92 & 8,84 & 2,84 & 7,63 & 17,34 & 3,30 & 18,56 & 4,01 & 17,02 & 3,58 \\
\hline Desvio padrão & 0,67 & 1,67 & 1,01 & 1,55 & 0,76 & 1,20 & 2,34 & 0,88 & 3,45 & 0,65 & 1,01 & 0,68 \\
\hline
\end{tabular}

Tabela 5. Composição dos resíduos poliméricos, pós-consumo - plásticos rígidos - por tipo de resina, gerados no município de São Carlos, SP, em percentual de massa.

\begin{tabular}{|c|c|c|c|c|c|c|}
\hline \multirow[t]{2}{*}{ Resina } & \multicolumn{2}{|c|}{ Inverno } & \multicolumn{2}{|c|}{ Verão } & \multicolumn{2}{|c|}{ Média Geral } \\
\hline & Média & Desvio-padrão & Média & Desvio padrão & Média & Desvio padrão \\
\hline \multicolumn{7}{|c|}{ Coleta regular } \\
\hline PET & 36,74 & 5,84 & 35,19 & 8,10 & 35,96 & 4,88 \\
\hline PEAD & 28,58 & 4,22 & 24,25 & 5,38 & 26,42 & 3,07 \\
\hline PVC & 3,76 & 2,85 & 3,77 & 3,42 & 3,76 & 2,30 \\
\hline PEBD & 1,69 & 2,27 & 1,89 & 1,09 & 1,79 & 1,31 \\
\hline PP & 17,36 & 3,36 & 15,14 & 3,87 & 16,25 & 2,78 \\
\hline PS & 6,29 & 3,91 & 9,64 & 6,37 & 7,96 & 4,71 \\
\hline Outros & 5,58 & 4,12 & 10,13 & 8,74 & 7,85 & 5,13 \\
\hline \multicolumn{7}{|c|}{ Coleta seletiva } \\
\hline PET & 52,81 & 9,85 & 48,91 & 11,91 & 50,64 & 6,44 \\
\hline PEAD & 23,80 & 5,17 & 22,74 & 5,69 & 24,03 & 5,40 \\
\hline PVC & 1,30 & 1,06 & 1,50 & 0,98 & 1,12 & 0,50 \\
\hline PEBD & 1,57 & 0,58 & 2,03 & 0,51 & 1,70 & 0,43 \\
\hline PP & 11,71 & 2,81 & 14,55 & 3,42 & 13,76 & 1,36 \\
\hline PS & 4,58 & 3,56 & 8,67 & 2,81 & 6,96 & 1,55 \\
\hline Outros & 4,23 & 3,69 & 1,59 & 1,45 & 1,79 & 1,01 \\
\hline
\end{tabular}


Tabela 6. Composição dos resíduos poliméricos, pós-consumo - plásticos rígidos - por tipo de uso, gerados no município de São Carlos, SP, em percentual de massa.

\begin{tabular}{|c|c|c|c|c|c|c|c|}
\hline Uso & & Refrigerante & Higiene Pessoal & Limpeza & Alimentos & Automotivo & Outros usos \\
\hline \multirow{9}{*}{$\begin{array}{c}\text { Coleta } \\
\text { regular }\end{array}$} & \multicolumn{7}{|c|}{ Inverno } \\
\hline & Média & 16,58 & 15,51 & 16,45 & 41,50 & 0,67 & 9,28 \\
\hline & $\begin{array}{l}\text { Desvio } \\
\text { padrão }\end{array}$ & 5,61 & 5,80 & 4,22 & 4,78 & 1,38 & 3,96 \\
\hline & \multicolumn{7}{|c|}{ Verão } \\
\hline & Média & 19,72 & 13,55 & 15,74 & 35,04 & 1,41 & 14,53 \\
\hline & $\begin{array}{l}\text { Desvio } \\
\text { padrão }\end{array}$ & 8,28 & 6,67 & 4,14 & 7,48 & 3,15 & 8,87 \\
\hline & \multicolumn{7}{|c|}{ Média geral } \\
\hline & Média & 18,15 & 14,53 & 16,10 & 38,27 & 1,04 & 11,90 \\
\hline & $\begin{array}{l}\text { Desvio } \\
\text { padrão }\end{array}$ & 4,16 & 4,65 & 2,77 & 4,69 & 2,10 & 5,56 \\
\hline \multirow{9}{*}{$\begin{array}{c}\text { Coleta } \\
\text { seletiva }\end{array}$} & \multicolumn{7}{|c|}{ Inverno } \\
\hline & Média & 27,90 & 9,27 & 18,91 & 36,15 & 1,35 & 6,42 \\
\hline & $\begin{array}{c}\text { Desvio } \\
\text { padrão }\end{array}$ & 5,71 & 1,33 & 2,42 & 5,28 & 0,42 & 4,42 \\
\hline & \multicolumn{7}{|c|}{ Verão } \\
\hline & Média & 30,76 & 8,08 & 18,22 & 35,28 & 1,66 & 5,99 \\
\hline & $\begin{array}{l}\text { Desvio } \\
\text { padrão }\end{array}$ & 7,94 & 1,77 & 2,64 & 2,78 & 0,68 & 2,03 \\
\hline & \multicolumn{7}{|c|}{ Média geral } \\
\hline & Média & 29,01 & 8,34 & 18,82 & 37,05 & 1,42 & 5,36 \\
\hline & $\begin{array}{l}\text { Desvio } \\
\text { padrão }\end{array}$ & 3,80 & 1,85 & 2,25 & 1,31 & 0,50 & 1,16 \\
\hline
\end{tabular}

Na caracterização dos resíduos sólidos poliméricos, pósconsumo - plásticos rígidos - da coleta regular, determinou-se uma composição, em massa, por tipo de resina, com o PET (35,96\%), PEAD (26,42\%), PP (16,25\%), PS (7,96\%), Outros $(7,85 \%)$, PVC $(3,76 \%)$, PEBD (1,79\%). Na coleta seletiva, determinou-se uma composição com o PET $(50,64 \%)$, PEAD $(24,03 \%)$, PP $(13,76 \%)$, PS (6,96\%), Outros $(1,79 \%)$, PEBD $(1,70 \%)$; PVC $(1,12 \%)$. Nos dois sistemas de coleta, somente os resíduos poliméricos com resinas de PET, PEAD e PP apresentaram desvios-padrão bem abaixo da média, indicando regularidade na geração. Nas duas coletas, observou-se, nos resíduos poliméricos de plástico rígido, o uso preferencial dos polímeros em embalagens de produtos alimentícios com percentual de participação, em massa, da ordem de 56,42\% na coleta regular e de $66,06 \%$ na coleta seletiva.

No verão os usos como alimentação, limpeza e higiene pessoal apresentaram decréscimos nos percentuais de participação, em relação ao inverno. O uso em refrigerante teve crescimento de $3,14 \%$, na coleta regular, e de $2,86 \%$, na coleta seletiva, em relação ao inverno, confirmando a tendência de consumo deste produto em estação quente. Os resíduos de polímeros usados em alimentação, refrigerante, limpeza e higiene pessoal apresentaram desvios-padrão abaixo da média, indicando regularidade na geração.

O PET é a resina de embalagens pós-consumo de maior participação percentual nas coletas, regular e seletiva, o que confirma a tendência de crescimento de geração já observada nos resultados obtidos com as caracterizações realizadas em
Araraquara e Botucatu, municípios do Estado de São Paulo, conforme Tabela 2.

No período de realização da caracterização dos resíduos poliméricos, pós-consumo, na coleta regular, registrou-se uma temperatura média de $15,8{ }^{\circ} \mathrm{C}$, no período de inverno, e de $21,3{ }^{\circ} \mathrm{C}$ no verão. No período de caracterização da coleta seletiva, a temperatura média de inverno foi de $17,5^{\circ} \mathrm{C}$ e a temperatura média de verão foi $23^{\circ} \mathrm{C}$. As diferenças de temperatura entre as estações tiveram registro de 5,5 e $6,0{ }^{\circ} \mathrm{C}$, nas coletas regular e seletiva, respectivamente. Os registros, em cada coleta, indicam que as temperaturas são muito próximas e que, entre as estações climáticas, as diferenças são suficientes para alterar os hábitos da população, evidenciado no crescimento do consumo de refrigerantes na estação do verão, nos dois sistemas de coleta, o que pode ser observado na Tabela 6.

\section{Conclusões}

Constatou-se que o poder público municipal de São Carlos, SP, se empenha em desenvolver política ambiental por meio de programas e ações para proteção do meio ambiente. É necessário, no entanto, minimizar os índices de resíduos poliméricos coletados e destinados ao aterro sanitário, o que, para tanto, recomenda-se a reestruturação do sistema de coleta seletiva, envolvendo ações como:

- Ampliação para $100 \%$ da área urbana e elevação da freqüência da coleta em cada bairro, por meio da cria- 
ção de novas cooperativas e aumento do número de cooperados;

- Ampliação do apoio logístico e organizacional para as cooperativas de catadores;

- Incentivo fiscal e apoio tecnológico para implantação de micro-empresas de reciclagem, com prioridade para população de baixa renda; e

- Campanhas publicitárias e valorização de iniciativas que visem à educação ambiental da população com foco na redução de desperdícios e na separação dos resíduos.

\section{Agradecimentos}

Pela parceria com outros projetos de pesquisa do NEPER - Núcleo de Estudo e Pesquisa em Resíduos Sólidos/SHS/ EESC/USP e à CAPES, pelo financiamento por meio do convênio PQI n. ${ }^{\circ}$ : 06/03-8.

\section{Referências Bibliográficas}

1. Matos, T. F. L. - "Diagnóstico dos resíduos poliméricos presentes nos resíduos domiciliares gerados em São Carlos, SP", Dissertação de Mestrado. Programa de Ciências da Engenharia Ambiental. Escola de Engenharia de São Carlos. Universidade de São Paulo. São Carlos/SP (2006).

2. Gomes, L. P. - "Estudo da caracterização física e da biodegrabilidade dos resíduos sólidos urbanos em aterros sanitários”, Dissertação de mestrado - Escola de Engenharia de São Carlos da Universidade de São Paulo. São Carlos/SP (1989).

3. Zeng, Y.; Trauth, K. M.; Peyton, R. L. \& Banerji, S. K. Waste Management \& Research, 23. p.62-71 (2005).

4. COMPANHIA DE LIMPEZA URBANA DO RIO DE JANEIRO - COMLURB - Relatório final do trabalho de caracterização dos resíduos Urbanos coletados nos domicílios da cidade do rio de janeiro. Rio de Janeiro (2002).
5. Zanin, M. \& Mancini, S. D. - "Resíduos plásticos e reciclagem: aspectos gerais e tecnologia", São Carlos/SP: EdUFSCar (2004).

6. INSTITUTO BRASILEIRO DE GEOGRAFIA E ESTATÍTICA - IBGE. Estimativas da população. Disponível em: http://ibge.gov.br. Acesso em 20.12.2005 (2005).

7. SECRETARIA MUNICIPAL DE DESENVOLVIMENTO SUSTENTÁVEL CIÊNCIA E TECNOLOGIA. Dados operacionais e de custos dos sistemas de coleta convencional e seletiva de São Carlos. Publicação eletrônica em Microsoft Excel. [Mensagem Pessoal]. Mensagem recebida por lofti@cefetce.br. Em 11/01/2005 (2005).

8. PREFEITURA MUNICIPAL DE SÃOCARLOS. PMSC. Secretaria Municipal de Desenvolvimento Sustentável Ciência E Tecnologia. Prospecto explicativo da coleta seletiva. São Carlos/SP (2004).

9. INSTITUTO DE PESQUISAS TECNOLÓGICAS DO ESTADO DE SÃO PAULO S.A.(IPT) / COMPROMISSO EMPRESARIAL PARA RECICLAGEM (CEMPRE), Lixo Municipal: Manual de Gerenciamento Integrado. Publicação IPT 2622, São Paulo/SP (2000).

10. ASSOCIAÇÃO BRASILEIRA DE NORMAS TÉCNICAS - NBR 10007: Amostragem de resíduos sólidos. Rio de Janeiro. (2004).

11. AMERICAN SOCIETY FOR TESTING AND MATERIALS. International. - ASTM D 5231-92 Standard Test Method for Determination of the Composition of Unprocessed Municipal Solid Waste. For referenced ASTM standards. (1992)

12. ASSOCIAÇÃO BRASILEIRA DE NORMAS TÉCNICAS. - NBR 13230: Simbologia indicativa de reciclabilidade e identificação de materiais plásticos - Rio de Janeiro (1994).

Enviado: 09/03/2007

Reenviado:10/05/2007 Aceito:17/05/07 